\title{
EHMTI-0318. The place of corticosteroids in migraine attack management: systematic review and critical appraisal
}

\author{
YW Woldeamanuel ${ }^{1 *}$, AM Rapoport ${ }^{2}$, RP Cowan ${ }^{1}$ \\ From 4th European Headache and Migraine Trust International Congress: EHMTIC 2014 \\ Copenhagen, Denmark. 18-21 September 2014
}

\section{Introduction}

Headaches recur in up to $87 \%$ of migraine patients visiting the emergency department (ED), making ED recidivism a management challenge.

\section{Aims}

We aimed herein to determine the role of corticosteroids in the acute management of migraine in the ED and outpatient care.

\section{Methods}

A PubMed search was employed for Clinical Studies and Systematic Reviews on the PubMed Clinical Queries tool combining the terms 'migraine' and 'corticosteroids' from 1980 until May 1, 2014.

\section{Results}

Twenty-two studies ( $\mathrm{n}=2203,50 \%$ ED-based, 64\% randomized-controlled) and four systematic reviews were included. International Classification of Headache Disorders criteria were applied in $68 \%$. Twenty-one studies indicated observed outcome differences favoring benefits of corticosteroid administration. Median absolute risk reduction was 30\% (range $6-48.2 \%)$ and $11 \%(6-48.6 \%)$ for 24- and 72-hour headache recurrence, respectively. Parenteral dexamethasone was the most commonly (65\%) administered steroid, at an average single dose of $12.8 \mathrm{mg}$ (range 4 - $24 \mathrm{mg}$ ). All meta-analyses revealed efficacy of adjuvant corticosteroids to various abortive medications indicating generalizability. Adverse effects were tolerable. Higher disability, status migrainosus, incomplete pain relief, and previous history of headache recurrence predicted outcome favourability.

\section{Conclusions}

Our literature review suggests that with corticosteroid treatment, recurrent headaches become milder than pretreated headaches and later respond to nonsteroidal therapy. Single-dose intravenous dexamethasone provides reasonable option for managing resistant, severe, or prolonged migraine attacks; recommendations include 6-8 administrations per year with follow up of adverse effects.

No conflict of interest.

\section{Authors' details}

${ }^{1}$ Neurology and Neurological Sciences, Stanford University School of Medicine, Stanford, USA. ${ }^{2}$ The David Geffen School of Medicine, University of California in Los Angeles, Los Angeles, USA.

Published: 18 September 2014

doi:10.1186/1129-2377-15-S1-G42

Cite this article as: Woldeamanuel et al:: EHMTI-0318. The place of corticosteroids in migraine attack management: systematic review and critical appraisal. The Journal of Headache and Pain 2014 15(Suppl 1):G42.

${ }^{1}$ Neurology and Neurological Sciences, Stanford University School of

Medicine, Stanford, USA

Full list of author information is available at the end of the article

(c) 2014 Woldeamanuel et al; licensee Springer. This is an Open Access article distributed under the terms of the Creative Commons Attribution License (http://creativecommons.org/licenses/by/2.0), which permits unrestricted use, distribution, and reproduction in any medium, provided the original work is properly cited. 